\title{
ANTEROGRADE PERCUTANEOUS TREATMENT OF LESSER METATARSAL FRACTURES: TECHNICAL DESCRIPTION AND CLINICAL RESULTS
}

Daniel Baumfeld, Benjamim Dutra Macedo', Caio Nery², Leonardo Elias Esper ${ }^{3}$, Marco Aurelio Baldo Filho ${ }^{3}$

\section{ABSTRACT}

Objective: The aim of this study was to evaluate the results obtained using the anterograde percutaneous fixation technique for treating shaft and neck fractures of the lesser metatarsals. Methods: We prospectively evaluated 14 patients between 2003 and 2008, taking into consideration the topography of the fracture, trauma mechanism, associated comorbidities and AOFAS score for the forefoot. Results: The anatomical region most affected was the metatarsal neck (79\%). Involvement of multiple metatarsals (53\%) was more common than isolated

\section{INTRODUCTION}

Lesser metatarsal fractures are common causes of pain and functional disability in the lower limb, especially by producing significant sequelae and deformities. Despite its high incidence ${ }^{(1)}$, these fractures have received little attention in the literature ${ }^{(2)}$. MB fractures represent $3-7 \%$ of all fractures of the body and $35 \%$ of fractures of the foot and have a rate of 75 new cases per 10,000 persons per year ${ }^{(3-5)}$.

They can be isolated, multiple or occur in combination with fracture-dislocations of the Lisfranc joint. Most MB fractures result from low-energy trauma, but the high-energy injuries or crushings have increased their incidence due to motorcycle accidents ${ }^{(6,7)}$.

MB fractures are divided according to their anatomical location into proximal metaphyseal, diaphyseal, cervical (neck), and cephalic (head).

Diaphyseal fractures are most commonly oblique, although they may present themselves in various patterns. fractures $(47 \%)$. Low-energy trauma $(79 \%)$ was more frequent than high-energy trauma (21\%). Female patients with diabetes had the worst postoperative functional results. There were no postoperative complications relating to the type of treatment instituted. Conclusion: The surgical technique presented was efficient for treating fractures of the lesser metatarsals, with a lower complication rate than shown by other established techniques in the literature.

Keywords - Metatarsus, Fractures, Bone; Fracture Fixation; Forefoot, Human

They are very important because of the shortening and the multiaxial deviations that they can produce ${ }^{(8)}$.

Distal fractures (neck and head) are often transverse or short oblique and deviations, when they occur, are predominantly in the plantar and lateral directions $^{(9)}$.

According to literature, the central MB fractures occur more frequently than those of the first MB and multiple fractures are more common than those that are isolated.

Due to its intrinsic stability, these fractures tend not present gross deviations. However, depending on the intensity and direction of the traumatic vectors, the central metatarsals can be dislocated conjointly ${ }^{(10,11)}$.

In general, $\mathrm{MB}$ fractures without deviations are treated conservatively. Fractures with small displacements in the frontal plane, without shortening or angulation, can also be treated conservatively ${ }^{(12,13)}$.

Fractures with displacement in the sagittal plane can lead to changes in load distribution under the

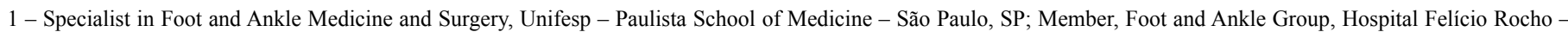
Belo Horizonte, MG, Brasil.

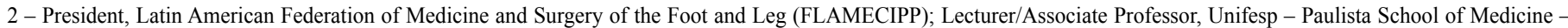
São Paulo, SP, Brasil.

3 - Third-Year Resident, Orthopedics and Traumatology, Hospital Felício Rocho, Belo Horizonte, MG, Brasil.

Study conducted at the Hospital Felício Rocho, Belo Horizonte, MG.

Correspondence: Rua Dr. Juvenal dos Santos, 325/401, Luxemburgo - 30380-530 - Belo Horizonte, MG. Email: danielbaumfeld@gmail.com

Received for publication: 2/12/2012, accepted for publication: 4/12/2012.

The authors declare that there was no conflict of interest in conducting this work 
metatarsal heads, resulting in painful calluses, mechanical metatarsalgias and traumatic neuroma formation $^{(13)}$. Shereff ${ }^{(14)}$ recommends reducing any fracture with a displacement of more than $3 \mathrm{~mm}$ in the frontal plane and an angle greater than 10 degrees in the sagittal plane (Table 1).

The surgical treatment established in the literature is fixation with Kirschner wires in a retrograde manner with exteriorization of the wire in the plantar region $^{(12,13,15,16)}$. Due to the high rate of complications related to this type of treatment, such as hypertrophic scars and painful calluses, besides metatarsophalangeal plantar plate lesions, we suggest a change in the direction to an antegrade introduction of the Kirschner wires.

The main objective of this study is to evaluate the results obtained with percutaneous antegrade fixation for fractures of the metatarsal diaphysis or neck, presenting the clinical and functional results after surgery.

Table 1 - Surgical indication for lesser metatarsal fractures according to Shereff ${ }^{(14)}$.

\begin{tabular}{c|c}
\hline Evaluation & Parameters \\
\hline Frontal plane & $>3$ to $4 \mathrm{~mm}$ of deviation \\
\hline Sagittal plane & Angulation $>10$ degrees \\
\hline Metatarsal formula & Changes in the metatarsal parabola \\
\hline
\end{tabular}

\section{MATERIALS AND METHODS}

The study protocol and informed consent forms were submitted and approved by the Research Ethics Committee of our hospital and the prospective collection of patients was initiated in 2003.

Fourteen patients with 26 surgical fractures of the lateral metatarsals classified according to the recommendations of Shereff presented in Table 1 were evaluated in the period between 2003 and 2008 .

Of these patients, eight were female and six were male. The average age at surgery was 39 years, ranging from 14 to 70 years.

Patients underwent standard clinical and radiological exams for their main complaint and responded to a questionnaire, through which information about the mechanism of injury, comorbidities, and lifestyle was collected.

Inclusion criteria for patients were:

Fractures of the lateral metatarsals (II to V) with surgical indication confirmed by radiographs of the feet "without load-bearing" in the anteroposterior, in- ternal oblique at 45 degrees, and profile views;

Absence of comorbidities that would prevent surgery, and

No other associated fractures.

For the postoperative clinical evaluation, we used the AOFAS functional score for the forefoot, obtained after six months of treatment.

\section{Surgical technique}

The patient is placed supine on a radiolucent table. With the aid of fluoroscopy, a small 5-mm surgical access is performed in the dorsal region of the foot, $10 \mathrm{~mm}$ from the base of the affected MB. With the aid of an acute drill, a small bony tunnel is excavated in the dorsal cortex of the MB until it reaches the medullary space. In this maneuver we take every care to preserve the plantar cortex of the metatarsal. Then, a Kirschner wire angled 15 degrees at its distal end is inserted anterogradely to the proximal edge of the fracture (Figure $1-\mathrm{A}-\mathrm{F}$ ). Fracture reduction is obtained by applying longitudinal traction combined with the manipulation of the forefoot. When closed reduction is impossible, a small incision in the area of the fracture allows the introduction of a delicate spatula to move the interposed tissues away and a bone forceps that assists in the alignment and reduction of the fracture (Figure 2). Through fluoroscopic vision, the remainder of the Kirschner wire is inserted until reaching the distal region of the $\mathrm{MB}$, and is kept $2 \mathrm{~mm}$ from the distal border of the head of the MB, avoiding its perforation (Figure $3-\mathrm{A}-\mathrm{F}$ ). The intramedullary wire acts as an internal tutor for maintaining reduction. Immediately after surgery, a plaster splint is applied for the purpose of analgesia and maintained for two weeks. After this time, a non-ambulatory boot is maintained for another four weeks. The Kirschner wire is removed at six weeks postoperatively. Physical therapy rehabilitation with gait training and range of motion gain is initiated as soon as the wire is removed, extending for another six weeks.

\section{RESULTS}

In all of the patients in this study, fracture healing was confirmed radiologically in the eighth week postoperatively.

Tables 2 and 3 show the percentage distribution of fractures according to their topography.

Table 4 presents the results according to the num- 

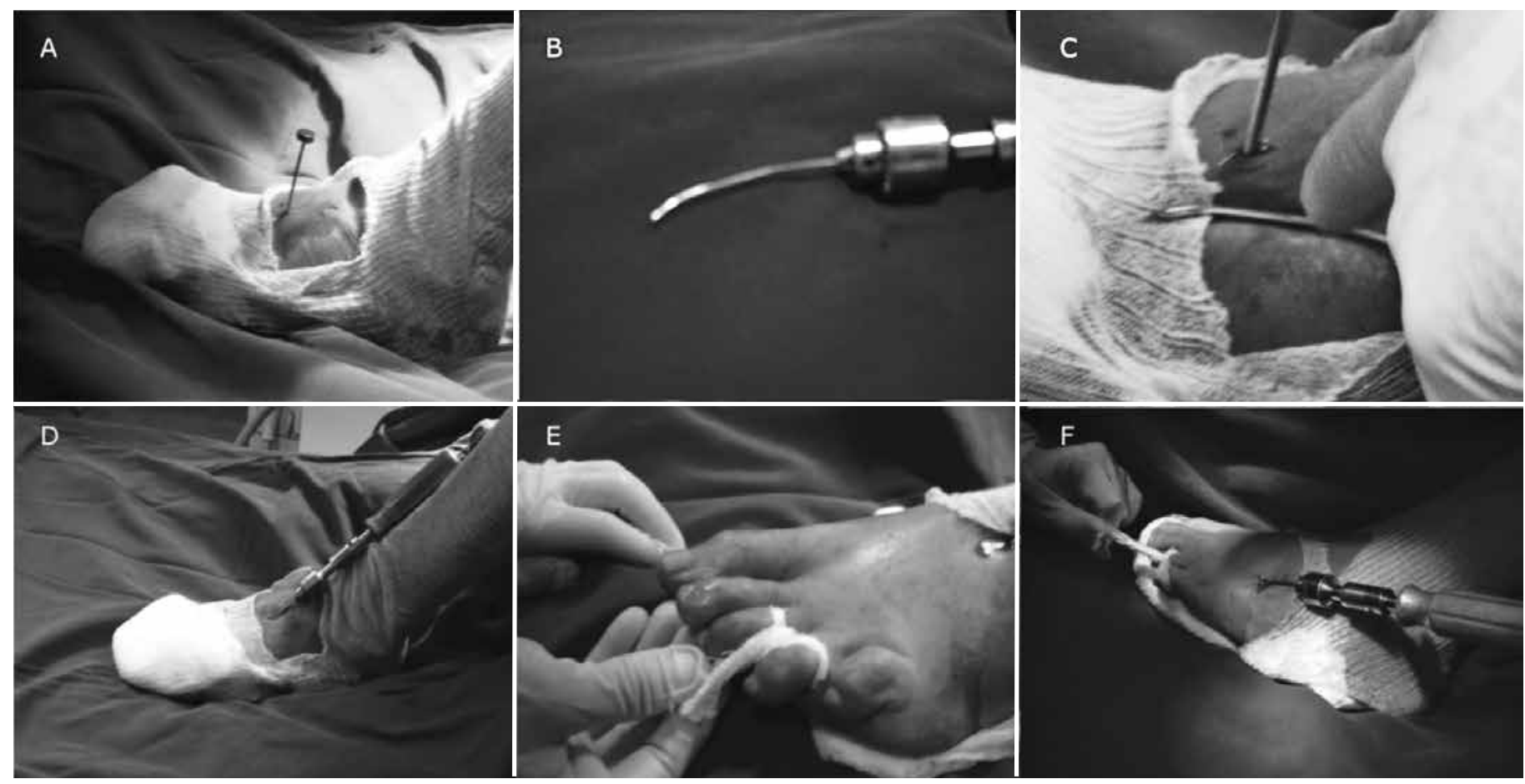

Figure 1 - Antegrade surgical technique. (A) Acute surgical drill introduced percutaneously $10 \mathrm{~mm}$ from the base of the fractured metatarsal. (B) Kirschner wire angled 15 degrees at its distal end. (C) Preparation for introducing the Kirschner wire in the intramedullary region of the metatarsus. (D) Kirschner wire inserted percutaneously. $(E)$ Longitudinal traction and manipulation of the forefoot to reduce the fracture. $(F)$ Kirschner wire inserted after fracture reduction.

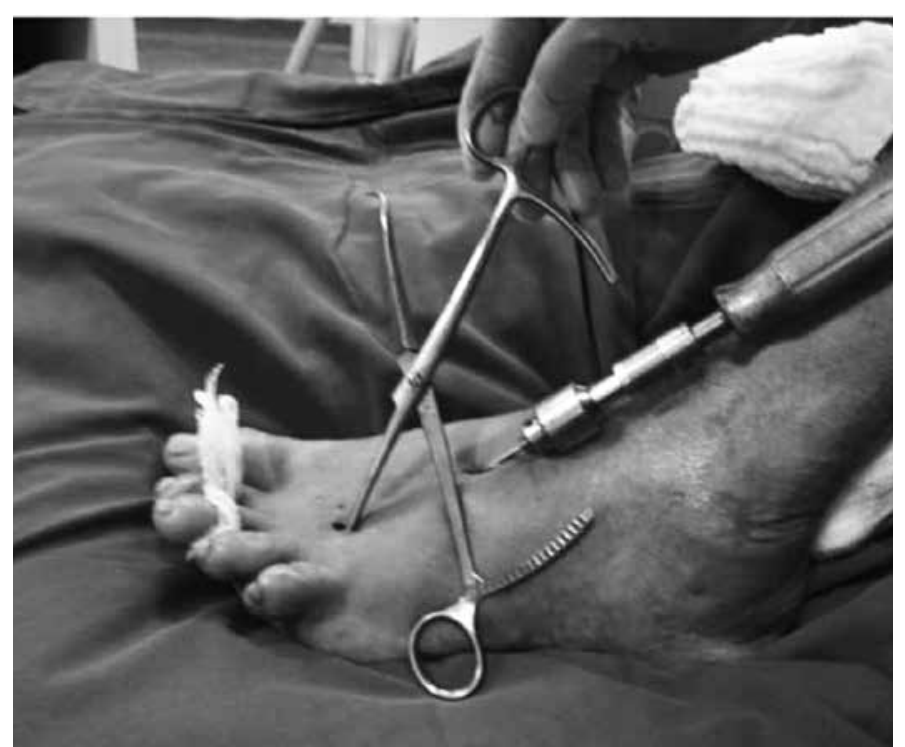

Figure 2 - Accessory incision to aid fracture reduction when the closed reduction could not be performed.

ber of metatarsals affected. The second MB was affected in isolation in $14 \%$ of patients, whereas in combination with other fractures, the second $\mathrm{MB}$ was fractured in $57 \%$ of patients.

Table 5 shows the overall incidence of fractured metatarsals. Table 6 shows the mechanism of injury of each patient studied. Table 7 shows the comorbidities found in the sample. Of the study patients, $21 \%$ (three patients) were smokers. The AOFAS score assessed at six months postoperatively averaged 98 points, ranging from 85 to 100 points.

Table 2 - Topography of the fractures and their incidence.

\begin{tabular}{c|c|c}
\hline Topography & $\mathbf{N}$ & $\%$ \\
\hline Neck & 20 & $77 \%$ \\
\hline Diaphysis & 6 & $23 \%$ \\
\hline Total & 26 & $100 \%$ \\
\hline
\end{tabular}

Table 3 - Anatomical location of fractures and their incidence.

\begin{tabular}{c|c|c|c|c}
\hline Topography & Pax & $\%$ & MBs & $\%$ \\
\hline Fracture of the neck of MB 2 & 2 & $14 \%$ & 2 & $10 \%$ \\
\hline Fracture of the neck of MB 3 & 1 & $7 \%$ & 1 & $5 \%$ \\
\hline Fracture of the necks of MBs 2 and 3 & 2 & $14 \%$ & 4 & $20 \%$ \\
\hline Fracture of the necks of MBs 2, 3 and 4 & 1 & $7 \%$ & 3 & $15 \%$ \\
\hline Fracture of the necks of MBs 2, 3, 4 and 5 & 1 & $7 \%$ & 4 & $20 \%$ \\
\hline Fracture of the necks of MBs 3 and 4 & 1 & $7 \%$ & 2 & $10 \%$ \\
\hline Fracture of the neck of MB 4 & 1 & $7 \%$ & 1 & $5 \%$ \\
\hline Fracture of the necks of MBs 4 and 5 & 1 & $7 \%$ & 2 & $10 \%$ \\
\hline Fracture of the neck of MB 5 & 1 & $7 \%$ & 1 & $5 \%$ \\
\hline Fractures of the neck & 11 & $79 \%$ & 20 & $77 \%$ \\
\hline Diaphyseal fracture of MBs 2 and 3 & 1 & $7 \%$ & 2 & $33 \%$ \\
\hline Diaphyseal fracture of MBs 2, 3 and 4 & 1 & $7 \%$ & 3 & $50 \%$ \\
\hline Diaphyseal fracture of MB 5 & 1 & $7 \%$ & 1 & $17 \%$ \\
\hline Diaphyseal fractures & 3 & $21 \%$ & 6 & $23 \%$ \\
\hline Total & 14 & $100 \%$ & 26 & $100 \%$ \\
\hline
\end{tabular}



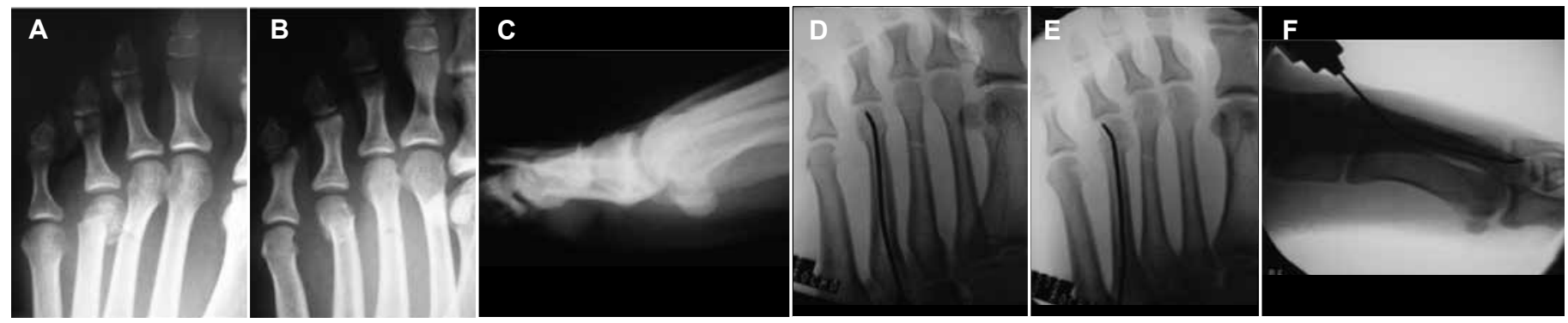

Figure 3 - Radiological demonstration of the percutaneous antegrade treatment. (A) Fracture of the neck of the fourth metatarsal with deviation greater than 3 mm in the frontal plane. (B) Deviation of the fracture in the oblique view of the foot. (C) Deviation of the fracture in the sagittal plane with more than 10 degrees of angulation. (D) Anteroposterior radiograph demonstrating reduction of the fracture and placement of intramedullary Kirschner wire in the fourth metatarsal $2 \mathrm{~mm}$ from the joint. (E) Demonstration of reduction and positioning of the wire in the oblique view of the foot. (F) Demonstration of wire positioning and reduction in lateral view.

Table 4 - Isolated impairment compared to multiple impairment.

\begin{tabular}{c|c|c}
\hline & N & $\%$ \\
\hline Impairment of multiple metatarsals & 8 & $57 \%$ \\
\hline Impairment of only one metatarsal & 6 & $43 \%$ \\
\hline Total & 14 & $100 \%$ \\
\hline
\end{tabular}

Table 5 - Anatomical location of the fractures and their percentages.

\begin{tabular}{c|c|c}
\hline Anatomical location & N & $\%$ \\
\hline Fracture of the neck of MB 2 & 6 & $43 \%$ \\
\hline Fracture of the neck of MB 3 & 6 & $43 \%$ \\
\hline Fracture of the neck of MB 4 & 5 & $36 \%$ \\
\hline Fracture of the neck of MB 5 & 3 & $21 \%$ \\
\hline Diaphyseal fracture MBs 2, 3, 4 & 1 & $7 \%$ \\
\hline Diaphyseal fracture MBs 2, 3 & 2 & $14 \%$ \\
\hline Diaphyseal fracture MB 5 & 1 & $7 \%$ \\
\hline
\end{tabular}

Table 6 - Trauma mechanism of the patients of this study.

\begin{tabular}{c|c|c}
\hline Mechanism of trauma & $\mathbf{N}$ & $\%$ \\
\hline Direct trauma & 5 & $36 \%$ \\
\hline Indirect trauma & 6 & $43 \%$ \\
\hline Traffic accident (motorcycle) & 3 & $21 \%$ \\
\hline Total & 14 & $100 \%$ \\
\hline
\end{tabular}

Table 7 - Comorbidities encountered in the patients of this study.

\begin{tabular}{c|c|c}
\hline Comorbidity & N & $\%$ \\
\hline Hypertension & 4 & $28 \%$ \\
\hline Diabetes & 2 & $14 \%$ \\
\hline
\end{tabular}

\section{DISCUSSION}

The MB fractures are among the most common injuries of the forefoot ${ }^{(3,4,6)}$. Its frequency is up to 10 times greater than the fractures affecting the Lisfranc joint ${ }^{(17)}$.

It is important to identify specific populations that are at risk of metatarsal fractures. These fractures are the most common forefoot fractures in motorcycle ac- cidents $^{(6)}$, but occur most commonly through low-energy trauma, resulting from direct trauma or simple twists ${ }^{(10)}$.

In our study, $69 \%$ of patients were low-energy trauma victims and $31 \%$ experienced high-energy trauma, conforming to the data found in the literature ${ }^{(3,7,10)}$.

The most widely used classification for these fractures is the topographic one, except for fractures located at the base of the fifth metatarsal ${ }^{(7)}$.

In this study, the most affected anatomical site was the neck of the second and third MBs with $43 \%$. More than one affected metatarsal represented $57 \%$ of our patients, which is also consistent with previous studies in the literature $e^{(1,10,11)}$.

Factors such as obesity, female sex, diabetes mellitus, and degree of deviation may worsen postoperative clinical outcomes ${ }^{(18)}$. Coincidentally, the only two patients in our study who had AOFAS scores below 100 points were female and diabetic, but these results were not statistically significant $(p>0.005)$. Smoking also was not a factor that changed the postoperative results $(\mathrm{p}>0.005)$.

Most metatarsal fractures are treated conservatively with or without plaster immobilization ${ }^{(19)}$. Surgical treatment is reserved for those fractures with more than 3-mm deviation or more than 10 degrees of angulation, due to the risk of metatarsalgia ${ }^{(20)}$.

If properly diagnosed and managed, these fractures have a good prognosis and low complication rates, but if not treated properly, they can lead to changes in gait and foot load distribution ${ }^{(1,11,18)}$.

The treatment recommended in the literature is retrograde fixation with Kirschner wires, opening the fracture site and exteriorizing the wire on the plantar surface of the foot ${ }^{(8,9,20)}$. The complications described 
in this type of treatment are painful plantar callus and metatarsophalangeal plantar plate injury ${ }^{(6,9,12,20)}$.

The patients in this study, treated by a percutaneous antegrade surgical approach, had higher AOFAS functional scores for the postoperative period, averaging more than 95 points, and no complications were identified related to the type of treatment used.

Despite the small number of patients in our sample, the established treatment proved sufficient to adequately treat metatarsal fractures, avoiding the postoperative complications of other treatments suggested in the literature.

\section{CONCLUSION}

Percutaneous antegrade surgical treatment is an effective alternative to other types of treatment for lateral metatarsal fractures, with a lower incidence of complications.

\section{REFERENCES}

1. Sánchez Alepuz E, Vicent Carsi V, Alcántara P, Llabrés AJ. Fractures of the central metatarsal. Foot Ankle Int. 1996;17(4):200-3.

2. Dobson R. The metatarsal finds stardom at last. BMJ. 2002;324(7343):933.

3. Court-Brown CM, Caesar B. Epidemiology of adult fractures: review. Injury. 2006;37(8):691-7.

4. Emmett JE, Breck LW. A review and analysis of 11,000 fractures seen in a private practice of orthopaedic surgery, 1937-1956. J Bone Joint Surg Am. 1958;40(5):1169-75.

5. Singer G, Cichocki M, Schalamon J, Eberl R, Höllwarth ME. A study of metatarsal fractures in children. J Bone Joint Surg Am. 2008;90(4):772-6.

6. Jeffers RF, Tan HB, Nicolopoulos C, Kamath R, Giannoudis PV. Prevalence and patterns of foot injuries following motorcycle trauma. J Orthop Trauma. 2004;18(2):87-91.

7. Rammelt S, Heineck J, Zwipp H. Metatarsal fractures. Injury. 2004;35(Suppl 2):SB77-86.

8. Maxwell JR. Open or closed treatment of metatarsal fractures. Indications and techniques. J Am Podiatry Assoc. 1983;73(2):100-6.

9. Heckman J. Fractures and dislocations of the foot. In: Rockwood C, Green D, editors. Fractures in adults. 2nd edition. Philadelphia: JB Lippincott; 1984. p. 1808-9.

10. Petrisor BA, Ekrol I, Court-Brown C. The epidemiology of metatarsal fractures. Foot Ankle Int. 2006;27(3):172-4.

11. Urteaga AJ, Lynch M. Fractures of the central metatarsals. Clin Podiatr Med Surg. 1995;12(4):759-72.

12. Sanders R.Fractures of the midfoot and forefoot. In: Mann RA, Coughlin MJ. Surgery of the foot and ankle. St Louis: Mosby; 2007. p. 1574-605.

13. Zwipp $\mathrm{H}$, Rammelt S. Frakturen und Luxationen. In: Wirth CJ. Orthopadie und Orthopadische Chirurgie. New York: Georg Thieme Verlag; 2002. p. 531-618.

14. Shereff MJ. Fractures of the forefoot. Instr Course Lect. 1990;39:133-40.

15. Heineck J, Liebscher T, Zwipp H. Fifth metatarsal base avulsion fractures. Orthop Traumatol; 2001:9:14-7.

16. Rettig AC, Shelbourne KD, Wilckens J. The surgical treatment of symptomatic nonunions of the proximal (metaphyseal) fifth metatarsal in athletes. Am J Sports Med. 1992;20(1):50-4.

17. Vuori JP, Aro HT. Lisfranc joint injuries: trauma mechanisms and associated injuries. J Trauma. 1993;35(1):40-5.

18. Cakir H, Van Vliet-Koppert ST, Van Lieshout EM, De Vries MR, Van Der Elst M, Schepers T. Demographics and outcome of metatarsal fractures. Arch Orthop Trauma Surg. 2011;131(2):241-5

19. Morrissey E Metatarsal fractures. J Bone Joint Surg Am; 1946,28:594-602.

20. Lee E, Donatto D. Fractures of the midfoot and forefoot. Curr Opin Orthop;1999; 10:224-30. 